\title{
Article
}

\section{Singularity Formation in the Inviscid Burgers Equation}

\author{
Giuseppe Maria Coclite $(\mathbb{D})$ 1,*(i) and Lorenzo di Ruvo ${ }^{2}$ \\ 1 Dipartimento di Meccanica, Matematica e Management, Politecnico di Bari, 70125 Bari, Italy \\ 2 Department of Mathematics, Università di Bari, 70125 Bari, Italy; lorenzo.diruvo@uniba.it \\ * Correspondence: giuseppemaria.coclite@poliba.it
}

check for updates

Citation: Coclite, G.M.; di Ruvo, L. Singularity Formation in the Inviscid Burgers Equation. Symmetry 2021, 13, 848. https://doi.org/10.3390/ sym 13050848

Academic Editor: Aviv Gibali

Received: 7 April 2021

Accepted: 2 May 2021

Published: 11 May 2021

Publisher's Note: MDPI stays neutral with regard to jurisdictional claims in published maps and institutional affiliations.

Copyright: (c) 2021 by the authors. Licensee MDPI, Basel, Switzerland. This article is an open access article distributed under the terms and conditions of the Creative Commons Attribution (CC BY) license (https:// creativecommons.org/licenses/by/ $4.0 /)$.

\begin{abstract}
We provide a lower bound for the blow up time of the $H^{2}$ norm of the entropy solutions of the inviscid Burgers equation in terms of the $H^{2}$ norm of the initial datum. This shows an interesting symmetry of the Burgers equation: the invariance of the space $H^{2}$ under the action of such nonlinear equation. The argument is based on a priori estimates of energy and stability type for the (viscous) Burgers equation.
\end{abstract}

Keywords: existence; uniqueness; stability; Burgers equation; Cauchy problem

MSC: 35G25; 35K55

\section{Introduction}

Consider the Cauchy problem for the inviscid Burgers equation:

$$
\begin{cases}\partial_{t} u+u \partial_{x} u=0, & 0<t<T, \quad x \in \mathbb{R}, \\ u(0, x)=u_{0}(x), & x \in \mathbb{R},\end{cases}
$$

and on the initial datum assume

$$
u_{0} \in H^{2}(\mathbb{R}), \quad u_{0} \neq 0 .
$$

Following the classical Kružkov approach [1], we use the following definition of solution.

Definition 1. A function $u:[0, T) \times \mathbb{R} \rightarrow \mathbb{R}$ is an entropy solution of (1) if

$$
u \in L_{l o c}^{\infty}((0, T) \times \mathbb{R})
$$

and for every constant $c \in \mathbb{R}$ and every nonnegative test function $\varphi \in C^{\infty}((-\infty, T) \times \mathbb{R})$ with compact support

$$
\int_{0}^{T} \int_{\mathbb{R}}\left(|u-c| \partial_{t} \varphi+\operatorname{sign}(u-c) \frac{u^{2}-c^{2}}{2} \partial_{x} \varphi\right) d t d x+\int_{\mathbb{R}}\left|u_{0}(x)-c\right| \varphi(0, x) d x \geq 0 .
$$

We know that, independently of the regularity assumptions on the initial datum, such as (2), the entropy solutions are unique and may develop a discontinuity in finite time (see [2-4]). On the other hand, the existence of smooth solutions of (1) is guaranteed for a short time by the Cauchy-Kovalevskaya Theorem [5]. Several papers on the development of singularities of nonlinear hyperbolic equations are available in the literature on this topic starting from the classical ones [6-8] and arriving at the more modern ones like [9-11] where tools of complex analysis are used and [12] where the time derivative is replaced by the Caputo one.

A very detailed blow-up analysis on the solutions of (1) can be carried using the characteristic lines of the equation for $\partial_{x} u$ that is 


$$
\partial_{t x}^{2} u+u \partial_{x}^{2} u+\left(\partial_{x} u\right)^{2}=0 .
$$

Indeed, in ([2] Example 1.4), choosing

$$
u_{0}(x)=\frac{1}{1+x^{2}}
$$

the author shows that the characteristic lines cross at time $T=8 / \sqrt{27}$ causing the creation of a shock.

A more refined tool that can be used for the analysis of the geometric structure and the large-time behavior of the solution of (1) is the Hopf-Cole [13-15] transformation that turns the (inviscid) Burgers equation into the linear heat equation. It provides a simple geometric construction of the solution, and allows to conclude that

- the initial information $u_{0}\left(x_{0}\right)$ propagates along the characteristic line $x=u_{0}\left(x_{0}\right) t_{0}$;

- the initial information that reaches a given location $(t, x)$ depends on the global minimum of the function

$$
F(t, x, y)=\frac{(x-y)^{2}}{2 t}+\int_{0}^{y} u_{0}(\xi) d \xi
$$

- a shock wave in (1) is experienced when the minimum of $F$ is attained in more than one location.

Here, we provide a lower bound for the maximal time $T$ of existence of an $H^{2}$ solution of (1) in terms of the $H^{2}$ norm of the initial datum. This result shows also a symmetry of the Burgers equations: the invariance of the space $\mathrm{H}^{2}$ under the action of that nonlinear equation in the time interval $[0, T]$. In addition, we also prove that as soon as the solution stays in $H^{2}$ it is also $L^{2}$ stable. It is interesting to note that, while the $L^{1}$ distance between entropy solutions of (1) is non-increasing, that is not the case for the $L^{2}$ distance. Our arguments are based on energy and stability estimates on the (viscous) Burgers equation.

The main result of this paper is the following theorem:

Theorem 1. Assume (2) and

$$
T<\frac{1}{3} \log \left(A_{0}\right), \quad \text { or } \quad\left\|\partial_{x} u_{0}\right\|_{H^{1}(\mathbb{R})}^{2}<\frac{1}{e^{3 T}-1},
$$

where

$$
A_{0}=\frac{\left\|\partial_{x} u_{0}\right\|_{H^{1}(\mathbb{R})}^{2}+1}{\left\|\partial_{x} u_{0}\right\|_{H^{1}(\mathbb{R})}^{2}} .
$$

There, the unique entropy solution $u$ of (1) in the sense of Definition 1 satisfies

$$
\begin{aligned}
& u \in H^{1}((0, T) \times \mathbb{R}) \cap L^{\infty}\left(0, T ; H^{2}(\mathbb{R})\right) \cap W^{1, \infty}((0, T) \times \mathbb{R}), \\
& \partial_{t} \partial_{x} u \in L^{2}(\mathbb{R}), \quad \text { for every } 0 \leq t \leq T .
\end{aligned}
$$

Moreover, if $u_{1}$ and $u_{2}$ are the two entropy solutions of (1) obtained in correspondence of the initial data $u_{1,0}$ and $u_{2,0}$ satisfying (3), the following stability estimate holds

$$
\left\|u_{1}(t, \cdot)-u_{2}(t, \cdot)\right\|_{L^{2}(\mathbb{R})}^{2} \leq e^{C t}\left\|u_{1,0}-u_{2,0}\right\|_{L^{2}(\mathbb{R})}^{2},
$$

for every $0 \leq t<T$, and for some positive constant $C$. 
Our argument is based on a priori estimates on the smooth solution $u_{\varepsilon}$ of the (viscous) Burgers equation of energy and stability type (see [16-18]):

$$
\begin{cases}\partial_{t} u_{\varepsilon}+u_{\varepsilon} \partial_{x} u_{\varepsilon}=\varepsilon \partial_{x}^{2} u_{\varepsilon}, & 0<t<T, \quad x \in \mathbb{R}, \\ u_{\varepsilon}(0, x)=u_{\varepsilon, 0}(x), & x \in \mathbb{R},\end{cases}
$$

where $0<\varepsilon<1$ and $u_{\varepsilon, 0}$ is an analytic approximation of $u_{0}$, such that

$$
\left\|u_{\varepsilon, 0}\right\|_{H^{2}(\mathbb{R})} \leq\left\|u_{0}\right\|_{H^{2}(\mathbb{R})}, \quad\left\|u_{\varepsilon, 0}\right\|_{L^{\infty}(\mathbb{R})} \leq\left\|u_{0}\right\|_{L^{\infty}(\mathbb{R})}, \quad \varepsilon\left\|\partial_{x}^{3} u_{\varepsilon, 0}\right\|_{L^{2}(\mathbb{R})}^{2} \leq C_{0}
$$

where $C_{0}$ an a positive constant independent of $\varepsilon$. Indeed we know that $[1,19]$

$$
u_{\varepsilon} \rightarrow u \quad \text { a.e. and in every } L_{l o c}^{p}((0, \infty) \times \mathbb{R}), 1 \leq p<\infty \text { ad } \varepsilon \rightarrow 0 .
$$

Thanks to the uniqueness of the entropy solution $u$ of (1) the limit is taken along all the family $\varepsilon \rightarrow 0$ and not only merely along a subsequence.

We obtain the regularity stated in (4), proving several energy estimates of $u_{\varepsilon}$ and using (4). Those are also the key tool for the proof of the stability estimate (5).

The proof of Theorem 1 is given in the next section.

\section{Proof of Theorem 1}

In what follows, we denote with $C$ all the positive constants independent on $\varepsilon$ and $t$.

The key tool in our argument is a precise analysis on the blow-up of the $H^{1}$ norm of the solution $u_{\varepsilon}$ of (6). For the sake fo readability we state and prove an $L^{\infty}$ and $L^{2}$ estimates on $u_{\varepsilon}$, that do not need assumption (3) (see [1]).

Lemma 1. Assume that (7) holds. For each $0<\varepsilon<1$, we have that

$$
\left\|u_{\varepsilon}\right\|_{L^{\infty}((0, T) \times \mathbb{R})} \leq\left\|u_{0}\right\|_{L^{\infty}(\mathbb{R})} \cdot
$$

for every $0 \leq t \leq T$.

Proof. Let $0 \leq t \leq T$. Observe that the constant maps $\left\|u_{0}\right\|_{L^{\infty}(\mathbb{R})}$ and $-\left\|u_{0}\right\|_{L^{\infty}(\mathbb{R})}$ solve the equation in (6). Therefore, is consequence of the comparison principle for parabolic equation and of the fact that (see (7))

$$
-\left\|u_{0}\right\|_{L^{\infty}(\mathbb{R})} \leq u_{0, \varepsilon} \leq\left\|u_{0}\right\|_{L^{\infty}(\mathbb{R})}
$$

that is (9).

Directly multiplying (6) by $u_{\varepsilon}$ we get the following $L^{2}$ estimate.

Lemma 2. Assume that (7) holds. We have that

$$
\left\|u_{\varepsilon}(t, \cdot)\right\|_{L^{2}(\mathbb{R})}^{2}+2 \varepsilon \int_{0}^{t}\left\|\partial_{x} u(s, \cdot)\right\|_{L^{2}(\mathbb{R})}^{2} d s \leq\left\|u_{0}\right\|_{L^{2}(\mathbb{R})}^{2}
$$

for every $0 \leq t \leq T$.

Proof. Let $0 \leq t \leq T$. Multiplying (6) by $2 u_{\varepsilon}$, an integration on $\mathbb{R}$ gives

$$
\begin{aligned}
\frac{d}{d t}\left\|u_{\varepsilon}(t, \cdot)\right\|_{L^{2}(\mathbb{R})}^{2} & =2 \int_{\mathbb{R}} u \partial_{t} u d x \\
& =-2 \int_{\mathbb{R}} u_{\varepsilon}^{2} \partial_{x} u_{\varepsilon} d x+2 \varepsilon \int_{\mathbb{R}} u_{\varepsilon} \partial_{x}^{2} u_{\varepsilon} d x=-2 \varepsilon\left\|\partial_{x} u_{\varepsilon}(t, \cdot)\right\|_{L^{2}(\mathbb{R})}^{2}
\end{aligned}
$$


Therefore, we have

$$
\frac{d}{d t}\left\|u_{\varepsilon}(t, \cdot)\right\|_{L^{2}(\mathbb{R})}^{2}+2 \varepsilon\left\|\partial_{x} u_{\varepsilon}(t, \cdot)\right\|_{L^{2}(\mathbb{R})}^{2}=0 .
$$

Integrating on $(0, t)$, thanks to $(7)$, we have (10).

In order to prove the invariance of the space $H^{2}$, we need to prove an a priori estate on the $H^{2}$-norm of $u_{\varepsilon}$ on a time interval independent on $\varepsilon$. As a first step in that direction we prove the following estimate that holds for any $t \geq 0$ and does not requires (3).

Lemma 3. Assume that (7) holds. We have that

$$
\frac{\left\|\partial_{x} u_{\varepsilon}(t, \cdot)\right\|_{H^{1}(\mathbb{R})}^{2}}{\left\|\partial_{x} u_{\varepsilon}(t, \cdot)\right\|_{H^{1}(\mathbb{R})}^{2}+1} \leq \frac{\left\|\partial_{x} u_{0}\right\|_{H^{1}(\mathbb{R})}^{2} e^{3 t}}{\left\|\partial_{x} u_{\varepsilon, 0}\right\|_{H^{1}(\mathbb{R})}^{2}+1}
$$

for every $t \geq 0$.

Proof. Multiplying (6) by $-2 \partial_{x}^{2} u_{\varepsilon}+2 \partial_{x}^{4} u_{\varepsilon}$, an integration on $\mathbb{R}$ gives

$$
\begin{aligned}
& \frac{d}{d t}\left(\left\|\partial_{x} u_{\varepsilon}(t, \cdot)\right\|_{L^{2}(\mathbb{R})}^{2}+\left\|\partial_{x}^{2} u_{\varepsilon}(t, \cdot)\right\|_{L^{2}(\mathbb{R})}^{2}\right) \\
& =-2 \int_{\mathbb{R}} \partial_{x}^{2} u_{\varepsilon} \partial_{t} u_{\varepsilon} d x+2 \int_{\mathbb{R}} \partial_{x}^{4} u_{\varepsilon} \partial_{t} u_{\varepsilon} d x \\
& =2 \int_{\mathbb{R}} u_{\varepsilon} \partial_{x} u_{\varepsilon} \partial_{x}^{2} u_{\varepsilon} d x-2 \int_{\mathbb{R}} u_{\varepsilon} \partial_{x} u_{\varepsilon} \partial_{x}^{4} u_{\varepsilon} d x-2 \varepsilon\left\|\partial_{x}^{2} u_{\varepsilon}(t, \cdot)\right\|_{L^{2}(\mathbb{R})}^{2}+2 \varepsilon \int_{\mathbb{R}} \partial_{x}^{2} u_{\varepsilon} \partial_{x}^{4} u_{\varepsilon} d x \\
& =-\int_{\mathbb{R}}\left(\partial_{x} u_{\varepsilon}\right)^{3} d x+2 \int_{\mathbb{R}}\left(\partial_{x} u_{\varepsilon}\right)^{2} \partial_{x}^{3} u_{\varepsilon} d x+2 \int_{\mathbb{R}} u_{\varepsilon} \partial_{x}^{2} u_{\varepsilon} \partial_{x}^{3} u_{\varepsilon} d x \\
& \quad-2 \varepsilon\left\|\partial_{x}^{2} u_{\varepsilon}(t, \cdot)\right\|_{L^{2}(\mathbb{R})}^{2}-2 \varepsilon\left\|\partial_{x}^{3} u_{\varepsilon}(t, \cdot)\right\|_{L^{2}(\mathbb{R})}^{2} \\
& =-\int_{\mathbb{R}}\left(\partial_{x} u_{\varepsilon}\right)^{3} d x-5 \int_{\mathbb{R}} \partial_{x} u_{\varepsilon}\left(\partial_{x}^{2} u_{\varepsilon}\right)^{2} d x-2 \varepsilon\left\|\partial_{x}^{2} u_{\varepsilon}(t, \cdot)\right\|_{L^{2}(\mathbb{R})}^{2}-2 \varepsilon\left\|\partial_{x}^{3} u_{\varepsilon}(t, \cdot)\right\|_{L^{2}(\mathbb{R})}^{2}
\end{aligned}
$$

Therefore, we have that

$$
\begin{aligned}
& \frac{d}{d t}\left(\left\|\partial_{x} u_{\varepsilon}(t, \cdot)\right\|_{L^{2}(\mathbb{R})}^{2}+\left\|\partial_{x}^{2} u_{\varepsilon}(t, \cdot)\right\|_{L^{2}(\mathbb{R})}^{2}\right) \\
& \quad+2 \varepsilon\left\|\partial_{x}^{2} u_{\varepsilon}(t, \cdot)\right\|_{L^{2}(\mathbb{R})}^{2}+2 \varepsilon\left\|\partial_{x}^{3} u_{\varepsilon}(t, \cdot)\right\|_{L^{2}(\mathbb{R})}^{2} \\
& \quad=-\int_{\mathbb{R}}\left(\partial_{x} u_{\varepsilon}\right)^{3} d x-5 \int_{\mathbb{R}} \partial_{x} u_{\varepsilon}\left(\partial_{x}^{2} u_{\varepsilon}\right)^{2} d x \\
& \quad \leq\left\|\partial_{x} u_{\varepsilon}(t, \cdot)\right\|_{L^{\infty}(\mathbb{R})}\left\|\partial_{x} u_{\varepsilon}(t, \cdot)\right\|_{L^{2}(\mathbb{R})}^{2}+5\left\|\partial_{x} u_{\varepsilon}(t, \cdot)\right\|_{L^{\infty}(\mathbb{R})}\left\|\partial_{x}^{2} u_{\varepsilon}(t, \cdot)\right\|_{L^{2}(\mathbb{R})}^{2} \\
& \quad \leq 6\left\|\partial_{x} u_{\varepsilon}(t, \cdot)\right\|_{L^{\infty}(\mathbb{R})}\left(\left\|\partial_{x} u_{\varepsilon}(t, \cdot)\right\|_{L^{2}(\mathbb{R})}^{2}+\left\|\partial_{x}^{2} u_{\varepsilon}(t, \cdot)\right\|_{L^{2}(\mathbb{R})}^{2}\right) .
\end{aligned}
$$

Define the following function

$$
X_{\varepsilon}(t):=\left\|\partial_{x} u_{\varepsilon}(t, \cdot)\right\|_{L^{2}(\mathbb{R})}^{2}+\left\|\partial_{x}^{2} u_{\varepsilon}(t, \cdot)\right\|_{L^{2}(\mathbb{R})}^{2}=\left\|\partial_{x} u_{\varepsilon}(t, \cdot)\right\|_{H^{1}(\mathbb{R})}^{2} \cdot
$$

Therefore, by (12),

$$
\frac{d X_{\varepsilon}(t)}{d t}+2 \varepsilon\left\|\partial_{x}^{2} u_{\varepsilon}(t, \cdot)\right\|_{L^{2}(\mathbb{R})}^{2}+2 \varepsilon\left\|\partial_{x}^{3} u_{\varepsilon}(t, \cdot)\right\|_{L^{2}(\mathbb{R})}^{2} \leq 6\left\|\partial_{x} u_{\varepsilon}(t, \cdot)\right\|_{L^{\infty}(\mathbb{R})} X_{\varepsilon}(t) .
$$


Due to the Young inequality,

$$
6\left\|\partial_{x} u_{\mathcal{\varepsilon}}(t, \cdot)\right\|_{L^{\infty}(\mathbb{R})} X_{\mathcal{E}}(t) \leq 3\left\|\partial_{x} u_{\mathcal{\varepsilon}}(t, \cdot)\right\|_{L^{\infty}(\mathbb{R})}^{2}+3 X_{\mathcal{\varepsilon}}^{2}(t) .
$$

It follows from (14) that

$$
\frac{d X_{\mathcal{\varepsilon}}(t)}{d t}+2 \varepsilon\left\|\partial_{x}^{2} u_{\varepsilon}(t, \cdot)\right\|_{L^{2}(\mathbb{R})}^{2}+2 \varepsilon\left\|\partial_{x}^{3} u_{\varepsilon}(t, \cdot)\right\|_{L^{2}(\mathbb{R})}^{2} \leq 3\left\|\partial_{x} u_{\varepsilon}(t, \cdot)\right\|_{L^{\infty}(\mathbb{R})}^{2}+3 X_{\varepsilon}^{2}(t) .
$$

Thanks to the Hölder inequality,

$$
\begin{aligned}
\left(\partial_{x} u_{\varepsilon}(t, x)\right)^{2} & =2 \int_{-\infty}^{x} \partial_{x} u_{\varepsilon} \partial_{x}^{2} u_{\varepsilon} d y \leq 2 \int_{\mathbb{R}}\left|\partial_{x} u_{\varepsilon}\right| \partial_{x}^{2} u_{\varepsilon} \mid d x \\
& \leq 2\left\|\partial_{x} u_{\varepsilon}(t, \cdot)\right\|_{L^{2}(\mathbb{R})}\left\|\partial_{x}^{2} u_{\varepsilon}(t, \cdot)\right\|_{L^{2}(\mathbb{R})} .
\end{aligned}
$$

Hence,

$$
\left\|\partial_{x} u_{\varepsilon}(t, \cdot)\right\|_{L^{\infty}(\mathbb{R})}^{2} \leq 2\left\|\partial_{x} u_{\varepsilon}(t, \cdot)\right\|_{L^{2}(\mathbb{R})}\left\|\partial_{x}^{2} u_{\varepsilon}(t, \cdot)\right\|_{L^{2}(\mathbb{R})} .
$$

Due to (13) and the Young inequality,

$$
\left\|\partial_{x} u_{\varepsilon}(t, \cdot)\right\|_{L^{\infty}(\mathbb{R})}^{2} \leq\left\|\partial_{x} u_{\varepsilon}(t, \cdot)\right\|_{L^{2}(\mathbb{R})}^{2}+\left\|\partial_{x}^{2} u_{\varepsilon}(t, \cdot)\right\|_{L^{2}(\mathbb{R})}^{2}=X_{\mathcal{\varepsilon}}(t)
$$

Consequently, by (15),

$$
\frac{d X_{\varepsilon}(t)}{d t}+2 \varepsilon\left\|\partial_{x}^{2} u_{\varepsilon}(t, \cdot)\right\|_{L^{2}(\mathbb{R})}^{2}+2 \varepsilon\left\|\partial_{x}^{3} u_{\varepsilon}(t, \cdot)\right\|_{L^{2}(\mathbb{R})}^{2} \leq 3 X_{\varepsilon}(t)\left(X_{\mathcal{\varepsilon}}(t)+1\right) .
$$

Then,

$$
\frac{d X_{\varepsilon}(t)}{d t} \leq 3 X_{\varepsilon}(t)\left(X_{\varepsilon}(t)+1\right)
$$

by (13),

$$
\frac{1}{X_{\mathcal{E}}(t)\left(X_{\mathcal{\varepsilon}}(t)+1\right)} \frac{d X_{\mathcal{\varepsilon}}(t)}{d t} \leq 3
$$

Integrating on $(0, t)$, we get

$$
\log \left(\frac{X_{\mathcal{\varepsilon}}(t)}{X_{\mathcal{\varepsilon}}(t)+1}\right)-\log \left(\frac{X_{0, \varepsilon}}{X_{0, \varepsilon}+1}\right) \leq 3 t
$$

Hence, thanks to (13),

$$
\log \left(\frac{\left\|\partial_{x} u_{\varepsilon}(t, \cdot)\right\|_{H^{1}(\mathbb{R})}^{2}}{\left\|\partial_{x} u_{\varepsilon}(t, \cdot)\right\|_{H^{1}(\mathbb{R})}^{2}+1}\right) \leq \log \left(\frac{\left\|\partial_{x} u_{0, \varepsilon}\right\|_{H^{1}(\mathbb{R})}^{2}}{\left\|\partial_{x} u_{0, \varepsilon}\right\|_{H^{1}(\mathbb{R})}^{2}+1}\right)+3 t
$$

which gives

$$
\frac{\left\|\partial_{x} u_{\varepsilon}(t, \cdot)\right\|_{H^{1}(\mathbb{R})}^{2}}{\left\|\partial_{x} u_{\varepsilon}(t, \cdot)\right\|_{H^{1}(\mathbb{R})}^{2}+1} \leq \frac{\left\|\partial_{x} u_{\varepsilon, 0}\right\|_{H^{1}(\mathbb{R})}^{2} e^{3 t}}{\left\|\partial_{x} u_{\varepsilon, 0}\right\|_{H^{1}(\mathbb{R})}^{2}+1}
$$

Using (7) in (18) we gain (11).

Using the assumption (3) on the time $T$, we are able to deduce for the previous general estimate the boudnedness of the family $\left\{u_{\varepsilon}\right\}_{\varepsilon}$ in the spaces $L^{\infty}\left(0, T ; H^{2}(\mathbb{R})\right)$ and $L^{\infty}\left(0, T ; W^{1, \infty}(\mathbb{R})\right)$. This is the core of our argument because it allows us to find that the entropy solution $u$ of (1) lives in the same spaces. 
Lemma 4. Assume that (3) and (7) hold. We have that

$$
\begin{aligned}
\frac{-1}{\left\|\partial_{x} u_{\varepsilon}(t, \cdot)\right\|_{H^{1}(\mathbb{R})}^{2}+1} & \leq \frac{-1}{\left\|\partial_{x} u_{\varepsilon, 0}\right\|_{H^{1}(\mathbb{R})}^{2}+1}+\frac{\left\|\partial_{x} u_{0}\right\|_{H^{1}(\mathbb{R})}^{2}}{\left\|\partial_{x} u_{\varepsilon, 0}\right\|_{H^{1}(\mathbb{R})}^{2}+1}\left(e^{3 t}-1\right), \\
\left\|\partial_{x} u_{\varepsilon}(t, \cdot)\right\|_{H^{1}(\mathbb{R})}^{2} & \leq \frac{\left\|\partial_{x} u_{0}\right\|_{H^{1}(\mathbb{R})}^{2} e^{3 T}}{\left[1-\left\|\partial_{x} u_{0}\right\|_{H^{1}(\mathbb{R})}^{2}\left(e^{3 T}-1\right)\right]^{3}},
\end{aligned}
$$

for every $0 \leq t \leq T$. In particular, we have

$$
\begin{array}{r}
\left\|\partial_{x} u_{\varepsilon}(t, \cdot)\right\|_{L^{2}(\mathbb{R})} \leq C \\
\left\|\partial_{x}^{2} u_{\varepsilon}(t, \cdot)\right\|_{L^{2}(\mathbb{R})} \leq C, \\
\left\|\partial_{x} u_{\varepsilon}\right\|_{L^{\infty}((0, T) \times \mathbb{R})} \leq C,
\end{array}
$$

for every $0 \leq t \leq T$ and some constant $C>0$, that depends on $u_{0}$ and $T$ but not on $\varepsilon$.

Proof. We begin by observing that, arguing as in Lemma 3, we have (17). Therefore, thanks to (11), (13) and (17),

$$
\begin{aligned}
\frac{d X_{\mathcal{\varepsilon}}(t)}{d t} & \leq \frac{3}{6} X_{\mathcal{\varepsilon}}(t)\left(X_{\mathcal{\varepsilon}}(t)+1\right) \\
& =\frac{3 X_{\mathcal{\varepsilon}}(t)}{X_{\mathcal{\varepsilon}}(t)+1}\left(X_{\mathcal{\varepsilon}}(t)+1\right)^{2} \leq \frac{3\left\|\partial_{x} u_{0}\right\|_{H^{1}(\mathbb{R})}^{2} e^{3 t}}{\left\|\partial_{x} u_{\varepsilon, 0}\right\|_{H^{1}(\mathbb{R})}^{2}+1}\left(X_{\mathcal{\varepsilon}}(t)+1\right)^{2}
\end{aligned}
$$

Therefore,

$$
\frac{1}{\left(X_{\varepsilon}(t)+1\right)^{2}} \frac{d X_{\varepsilon}(t)}{d t} \leq \frac{3\left\|\partial_{x} u_{0}\right\|_{H^{1}(\mathbb{R})}^{2} e^{3 t}}{\left\|\partial_{x} u_{\varepsilon, 0}\right\|_{H^{1}(\mathbb{R})}^{2}+1}
$$

Integrating on $(0, t)$, we have that

$$
\frac{-1}{X_{\varepsilon}(t)+1}+\frac{1}{X_{\varepsilon, 0}+1} \leq \frac{\left\|\partial_{x} u_{0}\right\|_{H^{1}(\mathbb{R})}^{2}}{\left\|\partial_{x} u_{\varepsilon, 0}\right\|_{H^{1}(\mathbb{R})}^{2}+1}\left(e^{3 t}-1\right)
$$

Thanks to (13), we get

$$
\frac{-1}{\left\|\partial_{x} u_{\varepsilon}(t, \cdot)\right\|_{H^{1}(\mathbb{R})}^{2}+1} \leq \frac{-1}{\left\|\partial_{x} u_{\varepsilon, 0}\right\|_{H^{1}(\mathbb{R})}^{2}+1}+\frac{\left\|\partial_{x} u_{0}\right\|_{H^{1}(\mathbb{R})}^{2}}{\left\|\partial_{x} u_{\varepsilon, 0}\right\|_{H^{1}(\mathbb{R})}^{2}+1}\left(e^{3 t}-1\right),
$$

which gives (19).

We conclude by proving (20). Thanks to (19), we have

$$
\frac{-1}{\left\|\partial_{x} u_{\varepsilon}(t, \cdot)\right\|_{H^{1}(\mathbb{R})}^{2}+1} \leq \frac{-1}{\left\|\partial_{x} u_{\varepsilon, 0}\right\|_{H^{1}(\mathbb{R})}^{2}+1}+\frac{\left\|\partial_{x} u_{0}\right\|_{H^{1}(\mathbb{R})}^{2}}{\left\|\partial_{x} u_{\varepsilon, 0}\right\|_{H^{1}(\mathbb{R})}^{2}+1}\left(e^{3 T}-1\right)
$$

Consequently, we obtain that

$$
\frac{\left[1-\left\|\partial_{x} u_{0}\right\|_{H^{1}(\mathbb{R})}^{2}\left(e^{3 T}-1\right)\right]}{\left\|\partial_{x} u_{\varepsilon, 0}\right\|_{H^{1}(\mathbb{R})}^{2}+1} \leq \frac{1}{\left\|\partial_{x} u_{\varepsilon}(t, \cdot)\right\|_{H^{1}(\mathbb{R})}^{2}+1} .
$$


Multiplying (24) by $\left\|\partial_{x} u_{\varepsilon}(t, \cdot)\right\|_{H^{1}(\mathbb{R})}^{2}$, we have that

$$
\frac{\left[1-\left\|\partial_{x} u_{0}\right\|_{H^{1}(\mathbb{R})}^{2}\left(e^{3 T}-1\right)\right]\left\|\partial_{x} u_{\varepsilon}(t, \cdot)\right\|_{H^{1}(\mathbb{R})}^{2}}{\left\|\partial_{x} u_{\varepsilon, 0}\right\|_{H^{1}(\mathbb{R})}^{2}+1} \leq \frac{\left\|\partial_{x} u_{\varepsilon}(t, \cdot)\right\|_{H^{1}(\mathbb{R})}^{2}}{\left\|\partial_{x} u_{\varepsilon}(t, \cdot)\right\|_{H^{1}(\mathbb{R})}^{2}+1} .
$$

Observe that by (11), we get

$$
\frac{\left\|\partial_{x} u_{\varepsilon}(t, \cdot)\right\|_{H^{1}(\mathbb{R})}^{2}}{\left\|\partial_{x} u_{\mathcal{\varepsilon}}(t, \cdot)\right\|_{H^{1}(\mathbb{R})}^{2}+1} \leq \frac{\left\|\partial_{x} u_{0}\right\|_{H^{1}(\mathbb{R})^{3 T}}^{2}}{\left\|\partial_{x} u_{\varepsilon, 0}\right\|_{H^{1}(\mathbb{R})}^{2}+1} .
$$

It follows from (25) and (26) that

$$
\frac{\left[1-\left\|\partial_{x} u_{0}\right\|_{H^{1}(\mathbb{R})}^{2}\left(e^{3 T}-1\right)\right]\left\|\partial_{x} u_{\varepsilon}(t, \cdot)\right\|_{H^{1}(\mathbb{R})}^{2}}{\left\|\partial_{x} u_{\varepsilon, 0}\right\|_{H^{1}(\mathbb{R})}^{2}+1} \leq \frac{\left\|\partial_{x} u_{0}\right\|_{H^{1}(\mathbb{R})}^{2} e^{3 T}}{\left\|\partial_{x} u_{\varepsilon, 0}\right\|_{H^{1}(\mathbb{R})}^{2}+1},
$$

that is

$$
\left[1-\left\|\partial_{x} u_{0}\right\|_{H^{1}(\mathbb{R})}^{2}\left(e^{3 T}-1\right)\right]\left\|\partial_{x} u_{\varepsilon}(t, \cdot)\right\|_{H^{1}(\mathbb{R})}^{2} \leq\left\|\partial_{x} u_{0}\right\|_{H^{1}(\mathbb{R})}^{2} e^{3 T} .
$$

Thanks to (3), we have that

$$
1-\left\|\partial_{x} u_{0}\right\|_{H^{1}(\mathbb{R})}^{2}\left(e^{3 T}-1\right)>0 .
$$

Therefore, by (27) and (28), we get

$$
\left\|\partial_{x} u_{\mathcal{\varepsilon}}(t, \cdot)\right\|_{H^{1}(\mathbb{R})}^{2} \leq \frac{\left\|\partial_{x} u_{0}\right\|_{H^{1}(\mathbb{R})}^{2} e^{3 T}}{\left[1-\left\|\partial_{x} u_{0}\right\|_{H^{1}(\mathbb{R})}^{2}\left(e^{3 T}-1\right)\right]^{2}}
$$

which gives (20).

Finally, (21)-(23) follows from (13), (16) and (20), respectively.

In order to prove the $L^{2}$-regularity of $\partial_{t} u$ we need the following energy estimate on the $H^{1}$ norm of $u_{\varepsilon}$.

Lemma 5. Assume that (3) and (7) hold. We have that

$$
\left\|\partial_{x} u_{\varepsilon}(t, \cdot)\right\|_{H^{1}(\mathbb{R})}^{2}+2 \varepsilon \int_{0}^{t}\left\|\partial_{x}^{2} u_{\varepsilon}(s, \cdot)\right\|_{H^{1}(\mathbb{R})}^{2} d s \leq C,
$$

for every $0 \leq t \leq T$ and some constant $C>0$, that depends on $u_{0}$ and $T$ but not on $\varepsilon$.

Proof. Let $0 \leq t \leq T$, where $T$ is defined in (3). Arguing as in Lemma 3, we (12). Therefore, by (12), (21)-(23), we get

$$
\begin{aligned}
& \frac{d}{d t}\left(\left\|\partial_{x} u_{\varepsilon}(t, \cdot)\right\|_{L^{2}(\mathbb{R})}^{2}+\left\|\partial_{x}^{2} u_{\varepsilon}(t, \cdot)\right\|_{L^{2}(\mathbb{R})}^{2}\right) \\
& \quad+2 \varepsilon\left\|\partial_{x}^{2} u_{\varepsilon}(t, \cdot)\right\|_{L^{2}(\mathbb{R})}^{2}+2 \varepsilon\left\|\partial_{x}^{3} u_{\varepsilon}(t, \cdot)\right\|_{L^{2}(\mathbb{R})}^{2} \\
& \quad \leq 6\left\|\partial_{x} u_{\varepsilon}(t, \cdot)\right\|_{L^{\infty}(\mathbb{R})}\left(\left\|\partial_{x} u_{\varepsilon}(t, \cdot)\right\|_{L^{2}(\mathbb{R})}^{2}+\left\|\partial_{x}^{2} u_{\varepsilon}(t, \cdot)\right\|_{L^{2}(\mathbb{R})}^{2}\right) \leq C .
\end{aligned}
$$

Integrating on $(0, t)$, by $(7)$, we have

$$
\left\|\partial_{x} u_{\mathcal{E}}(t, \cdot)\right\|_{L^{2}(\mathbb{R})}^{2}+\left\|\partial_{x}^{2} u_{\mathcal{\varepsilon}}(t, \cdot)\right\|_{L^{2}(\mathbb{R})}^{2}
$$




$$
\begin{aligned}
& \quad+2 \varepsilon \int_{0}^{t}\left\|\partial_{x}^{2} u_{\varepsilon}(s, \cdot)\right\|_{L^{2}(\mathbb{R})}^{2} d s+2 \varepsilon \int_{0}^{t}\left\|\partial_{x}^{3} u_{\varepsilon}(s, \cdot)\right\|_{L^{2}(\mathbb{R})}^{2} d s \\
& \leq\left\|u_{0}\right\|_{H^{2}(\mathbb{R})}+C t \leq C,
\end{aligned}
$$

which gives (29). on $\partial_{t} u_{\varepsilon}$.

We complete the proof of the $H^{1}$ regularity of $u$ proving the following bound $L^{2}$

Lemma 6. Assume that (3) and (7) hold. We have that

$$
\left\|\partial_{t} u_{\varepsilon}(t, \cdot)\right\|_{L^{2}(\mathbb{R})}^{2} \leq C,
$$

for every $0 \leq t \leq T$ and some constant $C>0$, that depends on $u_{0}$ and $T$ but not on $\varepsilon$.

Proof. Let $0 \leq t \leq T$. We begin by observing that, by (6),

$$
\left(\partial_{t} u_{\varepsilon}\right)^{2}=\left(-u_{\varepsilon} \partial_{x} u_{\varepsilon}+\varepsilon \partial_{x}^{2} u_{\varepsilon}\right)^{2}=u_{\varepsilon}^{2}\left(\partial_{x} u_{\varepsilon}\right)^{2}-2 \varepsilon u_{\varepsilon} \partial_{x} u_{\varepsilon} \partial_{x}^{2} u_{\varepsilon}+\varepsilon^{2}\left(\partial_{x}^{2} u_{\varepsilon}\right)^{2} .
$$

Since

$$
-2 \varepsilon \int_{\mathbb{R}} u_{\varepsilon} \partial_{x} u_{\varepsilon} \partial_{x}^{2} u_{\varepsilon} d x=\varepsilon \int_{\mathbb{R}}\left(\partial_{x} u_{\varepsilon}\right)^{3} d x,
$$

integrating (31) on $\mathbb{R}$, we have

$$
\left\|\partial_{t} u_{\varepsilon}(t, \cdot)\right\|_{L^{2}(\mathbb{R})}^{2}=\int_{\mathbb{R}} u_{\varepsilon}^{2}\left(\partial_{x} u_{\varepsilon}\right)^{2} d x+\varepsilon \int_{\mathbb{R}}\left(\partial_{x} u_{\varepsilon}\right)^{3} d x+\varepsilon^{2}\left\|\partial_{x}^{2} u_{\varepsilon}(t, \cdot)\right\|_{L^{2}(\mathbb{R})}^{2} .
$$

Since $0<\varepsilon<1$, due to (9), (21) and (22),

$$
\begin{aligned}
& \int_{\mathbb{R}} u_{\varepsilon}^{2}\left(\partial_{x} u_{\varepsilon}\right)^{2} d x \leq\left\|u_{\varepsilon}\right\|_{L^{\infty}((0, T) \times \mathbb{R})}^{2}\left\|\partial_{x} u_{\varepsilon}(t, \cdot)\right\|_{L^{2}(\mathbb{R})}^{2} \leq C, \\
& \varepsilon \int_{\mathbb{R}}\left|\partial_{x} u_{\varepsilon}\right|^{3} d x \leq \varepsilon\left\|\partial_{x} u_{\varepsilon}(t, \cdot)\right\|_{L^{\infty}((0, T) \times \mathbb{R})}\left\|\partial_{x} u_{\varepsilon}(t, \cdot)\right\|_{L^{2}(\mathbb{R})}^{2} \leq C, \\
& \varepsilon^{2}\left\|\partial_{x}^{2} u_{\varepsilon}(t, \cdot)\right\|_{L^{2}(\mathbb{R})}^{2} \leq C .
\end{aligned}
$$

Hence, by (32),

$$
\left\|\partial_{t} u_{\varepsilon}(t, \cdot)\right\|_{L^{2}(\mathbb{R})} \leq C,
$$

which gives (30).

In order to prove the $L^{2}$-regularity of $\partial_{t x}^{2} u$, we need the following energy estimate on the $H^{1}$ norm of $u_{\varepsilon}$.

Lemma 7. Assume that (3) and (7) hold. We have that

$$
\varepsilon\left\|\partial_{x}^{3} u_{\mathcal{\varepsilon}}(t, \cdot)\right\|_{L^{2}(\mathbb{R})}^{2}+2 \varepsilon^{2} \int_{0}^{t}\left\|\partial_{x}^{4} u_{\varepsilon}(s, \cdot)\right\|_{L^{2}(\mathbb{R})}^{2} d s \leq C,
$$

for every $0 \leq t \leq T$ and some constant $C>0$, that depends on $u_{0}$ and $T$ but not on $\varepsilon$.

Proof. Let $0 \leq t \leq T$. Multiplying (6), by $-2 \varepsilon \partial_{x}^{6} u_{\varepsilon}$, we have that

$$
\begin{aligned}
& -2 \varepsilon \int_{\mathbb{R}} \partial_{x}^{6} u_{\varepsilon} \partial_{t} u_{\varepsilon} d x=\varepsilon \frac{d}{d t}\left\|\partial_{x}^{3} u_{\varepsilon}(t, \cdot)\right\|_{L^{2}(\mathbb{R})}^{2} \\
& \quad=2 \varepsilon \int_{\mathbb{R}} u_{\varepsilon} \partial_{x} u_{\varepsilon} \partial_{x}^{6} u_{\varepsilon} d x+2 \varepsilon^{2} \int_{\mathbb{R}} \partial_{x}^{2} u_{\varepsilon} \partial_{x}^{6} u_{\varepsilon} d x \\
& \quad=-2 \varepsilon \int_{\mathbb{R}}\left(\partial_{x} u_{\varepsilon}\right)^{2} \partial_{x}^{5} u_{\varepsilon} d x-2 \varepsilon \int_{\mathbb{R}} u_{\varepsilon} \partial_{x}^{2} u_{\varepsilon} \partial_{x}^{5} u_{\varepsilon} d x-2 \varepsilon^{2} \int_{\mathbb{R}} \partial_{x}^{3} u_{\varepsilon} \partial_{x}^{5} u_{\varepsilon} d x
\end{aligned}
$$




$$
\begin{aligned}
& =6 \varepsilon \int_{\mathbb{R}} \partial_{x} u_{\varepsilon} \partial_{x}^{2} u_{\varepsilon} \partial_{x}^{4} u_{\varepsilon} d x+2 \varepsilon \int_{\mathbb{R}} u_{\varepsilon} \partial_{x}^{3} u_{\varepsilon} \partial_{x}^{4} u_{\varepsilon} d x-2 \varepsilon^{2}\left\|\partial_{x}^{4} u_{\varepsilon}(t, \cdot)\right\|_{L^{2}(\mathbb{R})}^{2} \\
& =-7 \varepsilon \int_{\mathbb{R}} \partial_{x} u_{\varepsilon}\left(\partial_{x}^{3} u_{\varepsilon}\right)^{2}+2 \varepsilon^{2}\left\|\partial_{x}^{4} u_{\varepsilon}(t, \cdot)\right\|_{L^{2}(\mathbb{R})}^{2} .
\end{aligned}
$$

Therefore, we have that

$$
\varepsilon \frac{d}{d t}\left\|\partial_{x}^{3} u_{\varepsilon}(t, \cdot)\right\|_{L^{2}(\mathbb{R})}^{2}+2 \varepsilon^{2}\left\|\partial_{x}^{4} u_{\varepsilon}(t, \cdot)\right\|_{L^{2}(\mathbb{R})}^{2}=-7 \varepsilon \int_{\mathbb{R}} \partial_{x} u_{\varepsilon}\left(\partial_{x}^{3} u_{\varepsilon}\right)^{2} d x
$$

Due to (23),

$$
7 \varepsilon \int_{\mathbb{R}}\left|\partial_{x} u_{\varepsilon}\right|\left(\partial_{x}^{3} u_{\varepsilon}\right)^{2} d x \leq \varepsilon\left\|\partial_{x} u_{\varepsilon}\right\|_{L^{\infty}((0, T) \times \mathbb{R})} \leq \varepsilon C\left\|\partial_{x}^{3} u_{\varepsilon}(t, \cdot)\right\|_{L^{2}(\mathbb{R})}^{2} .
$$

Consequently, by (34),

$$
\varepsilon \frac{d}{d t}\left\|\partial_{x}^{3} u_{\varepsilon}(t, \cdot)\right\|_{L^{2}(\mathbb{R})}^{2}+2 \varepsilon^{2}\left\|\partial_{x}^{4} u_{\mathcal{\varepsilon}}(t, \cdot)\right\|_{L^{2}(\mathbb{R})}^{2} \leq \varepsilon C\left\|\partial_{x}^{3} u_{\varepsilon}(t, \cdot)\right\|_{L^{2}(\mathbb{R})}^{2} .
$$

Integrating on $(0, t)$, by (7) and (29), we have that

$$
\varepsilon\left\|\partial_{x}^{3} u_{\varepsilon}(t, \cdot)\right\|_{L^{2}(\mathbb{R})}^{2}+2 \varepsilon^{2} \int_{0}^{t}\left\|\partial_{x}^{4} u_{\varepsilon}(s, \cdot)\right\|_{L^{2}(\mathbb{R})}^{2} d s \leq C_{0}+C \varepsilon \int_{0}^{t}\left\|\partial_{x}^{3} u_{\varepsilon}(s, \cdot)\right\|_{L^{2}(\mathbb{R})}^{2} d s \leq C,
$$

which gives (33).

In the followong final lemma we prove an $L^{2}$ bound on the mixed derivative $\partial_{t} \partial_{x} u_{\varepsilon}$, that implies an $L^{\infty}$ bound on the time derivative $\partial_{t} u_{\varepsilon}$.

Lemma 8. Assume that (3) and (7) hold. We have that

$$
\begin{aligned}
\left\|\partial_{t} \partial_{x} u_{\varepsilon}(t, \cdot)\right\|_{L^{2}(\mathbb{R})} & \leq C, \\
\left\|\partial_{t} u_{\varepsilon}\right\|_{L^{\infty}((0, T) \times \mathbb{R})} & \leq C,
\end{aligned}
$$

for every $0 \leq t \leq T$ and some constant $C>0$, that depends on $u_{0}$ and $T$ but not on $\varepsilon$.

Proof. Let $0 \leq t \leq T$, where $T$ is defined in (3). Multiplying (6) by $-2 \partial_{t} \partial_{x}^{2} u_{\varepsilon}$, we have that

$$
-2 \int_{\mathbb{R}} \partial_{t} \partial_{x}^{2} u_{\varepsilon} \partial_{t} u_{\varepsilon} d x=2 \int_{\mathbb{R}} u_{\varepsilon} \partial_{x} u_{\varepsilon} \partial_{t} \partial_{x}^{2} u_{\varepsilon} d x-2 \varepsilon \int_{\mathbb{R}} \partial_{x}^{2} u_{\varepsilon} \partial_{t} \partial_{x}^{2} u_{\varepsilon} d x
$$

Since

$$
\begin{aligned}
-2 \int_{\mathbb{R}} \partial_{t} \partial_{x}^{2} u_{\varepsilon} \partial_{t} u_{\varepsilon} d x & =2\left\|\partial_{t} \partial_{x} u_{\varepsilon}(t, \cdot)\right\|_{L^{2}(\mathbb{R})}^{2}, \\
2 \int_{\mathbb{R}} u_{\varepsilon} \partial_{x} u_{\varepsilon} \partial_{t} \partial_{x}^{2} u_{\varepsilon} & =-2 \int_{\mathbb{R}}\left(\partial_{x} u_{\varepsilon}\right)^{2} \partial_{t} \partial_{x} u_{\varepsilon} d x-2 \int_{\mathbb{R}} u_{\varepsilon} \partial_{x}^{2} u_{\varepsilon} \partial_{t} \partial_{x} u_{\varepsilon} d x, \\
2 \varepsilon \int_{\mathbb{R}} \partial_{x}^{2} u_{\varepsilon} \partial_{t} \partial_{x}^{2} u_{\varepsilon} d x & =-2 \varepsilon \int_{\mathbb{R}} \partial_{x}^{3} u_{\varepsilon} \partial_{t} \partial_{x} u_{\varepsilon} d x
\end{aligned}
$$

an integration of (37) on $\mathbb{R}$ gives

$$
\begin{aligned}
2\left\|\partial_{t} \partial_{x} u_{\varepsilon}(t, \cdot)\right\|_{L^{2}(\mathbb{R})}^{2}= & -2 \int_{\mathbb{R}}\left(\partial_{x} u_{\varepsilon}\right)^{2} \partial_{t} \partial_{x} u_{\varepsilon} d x-2 \int_{\mathbb{R}} u_{\varepsilon} \partial_{x}^{2} u_{\varepsilon} \partial_{t} \partial_{x} u_{\varepsilon} d x \\
& -2 \varepsilon \int_{\mathbb{R}} \partial_{x}^{3} u_{\varepsilon} \partial_{t} \partial_{x} u_{\varepsilon} d x
\end{aligned}
$$


Since $0<\varepsilon<1$, thanks to (9), (21)-(23), (33) and the Young inequality,

$$
\begin{gathered}
2 \int_{\mathbb{R}}\left(\partial_{x} u_{\varepsilon}\right)^{2}\left|\partial_{t} \partial_{x} u_{\varepsilon}\right| d x \leq 2\left\|\partial_{x} u_{\varepsilon}\right\|_{L^{\infty}(\mathbb{R})} \int_{\mathbb{R}}\left|\partial_{x} u_{\varepsilon}\right|\left|\partial_{t} \partial_{x} u_{\varepsilon}\right| d x \\
\leq 2 C \int_{\mathbb{R}}\left|\partial_{x} u_{\varepsilon}\right|\left|\partial_{t} \partial_{x} u_{\varepsilon}\right| d x \leq \int_{\mathbb{R}}\left|2 C \partial_{x} u_{\varepsilon}\right|\left|\partial_{t} \partial_{x} u_{\varepsilon}\right| d x \\
\leq C\left\|\partial_{x} u_{\varepsilon}(t, \cdot)\right\|_{L^{2}(\mathbb{R})}^{2}+\frac{1}{2}\left\|\partial_{t} \partial_{x} u_{\varepsilon}(t, \cdot)\right\|_{L^{2}(\mathbb{R})}^{2} \\
\leq C+\frac{1}{2}\left\|\partial_{t} \partial_{x} u_{\varepsilon}(t, \cdot)\right\|_{L^{2}(\mathbb{R})}^{2}, \\
2 \int_{\mathbb{R}}\left|u_{\varepsilon}\left\|\partial_{x}^{2} u_{\varepsilon}\right\| \partial_{t} \partial_{x} u_{\varepsilon}\right| d x \leq 2\left\|u_{\varepsilon}\right\|_{L^{\infty}((0, T) \times \mathbb{R})} \int_{\mathbb{R}}\left|\partial_{x}^{2} u_{\varepsilon} \| \partial_{t} \partial_{x} u_{\varepsilon}\right| d x \\
\leq 2\left\|u_{0}\right\|_{L^{\infty}(\mathbb{R})} \int_{\mathbb{R}}\left|\partial_{x}^{2} u_{\varepsilon}\left\|\partial_{t} \partial_{x} u_{\varepsilon}\left|d x=\int_{\mathbb{R}}\right| 2\right\| u_{0} \|_{L^{\infty}(\mathbb{R})} \partial_{x}^{2} u_{\varepsilon}\right|\left|\partial_{t} \partial_{x} u_{\varepsilon}\right| d x \\
\leq 2\left\|u_{0}\right\|_{L^{\infty}(\mathbb{R})}^{2}\left\|\partial_{x}^{2} u_{\varepsilon}(t, \cdot)\right\|_{L^{2}(\mathbb{R})}^{2}+\frac{1}{2}\left\|\partial_{t} \partial_{x} u_{\varepsilon}(t, \cdot)\right\|_{L^{2}(\mathbb{R})}^{2} \\
\leq C+\frac{1}{2}\left\|\partial_{t} \partial_{x} u_{\varepsilon}(t, \cdot)\right\|_{L^{2}(\mathbb{R})}^{2}, \\
2|\varepsilon| \int_{\mathbb{R}}\left|\partial_{x}^{3} u_{\varepsilon}\left\|\partial_{t} \partial_{x} u_{\varepsilon} \mid d x \leq 2 \varepsilon^{2}\right\| \partial_{x}^{3} u_{\varepsilon}(t, \cdot)\left\|_{L^{2}(\mathbb{R})}^{2}+\frac{1}{2}\right\| \partial_{t} \partial_{x} u_{\varepsilon}(t, \cdot) \|_{L^{2}(\mathbb{R})}^{2}\right. \\
\leq C+\frac{1}{2}\left\|\partial_{t} \partial_{x} u_{\varepsilon}(t, \cdot)\right\|_{L^{2}(\mathbb{R}) \cdot}^{2} \cdot
\end{gathered}
$$

Consequently, by (38),

$$
\frac{1}{2}\left\|\partial_{t} \partial_{x} u_{\mathcal{E}}(t, \cdot)\right\|_{L^{2}(\mathbb{R})}^{2} \leq C,
$$

which gives (35).

Finally, we prove (36). Thanks to (30) and (35) and the Hölder inequality,

$$
\begin{aligned}
\left(\partial_{t} u_{\varepsilon}(t, x)\right)^{2} & =2 \int_{-\infty}^{x} \partial_{t} u_{\varepsilon} \partial_{t} \partial_{x} u_{\varepsilon} d y \leq 2 \int_{\mathbb{R}}\left|\partial_{t} u_{\varepsilon} \| \partial_{t} \partial_{x} u_{\varepsilon}\right| d x \\
& \leq 2\left\|\partial_{t} u_{\varepsilon}(t, \cdot)\right\|_{L^{2}(\mathbb{R})}\left\|\partial_{t} \partial_{x} u_{\varepsilon}(t, \cdot)\right\|_{L^{2}(\mathbb{R})} \leq C .
\end{aligned}
$$

Hence,

$$
\left\|\partial_{t} u_{\mathcal{\varepsilon}}\right\|_{L^{\infty}((0, T) \times \mathbb{R})}^{2} \leq C,
$$

which gives (36).

We are ready for the proof of Theorem 1 .

Proof of Theorem 1. The convergence stated in (8) and the bounds proved in the previous lemmas gives the regularity on $u$ claimed in (4).

We prove (5). Let $u_{1}$ and $u_{2}$ be two solutions of (1), which verify (4), that is

$$
\begin{gathered}
\begin{cases}\partial_{t} u_{1}+\partial_{x} u_{1}=0, & 0<t<T, x \in \mathbb{R}, \\
u_{1}(0, x)=u_{1,0}(x), & x \in \mathbb{R},\end{cases} \\
\begin{cases}\partial_{t} u_{2}+u_{2} \partial_{x} u_{2}=0, & 0<t<T, x \in \mathbb{R}, \\
u_{2}(0, x)=u_{2,0}(x), & x \in \mathbb{R} .\end{cases}
\end{gathered}
$$

Then, the function

$$
\omega=u_{1}-u_{2}
$$

is the solution of the following Cauchy problem:

$$
\begin{cases}\partial_{t} \omega+u_{1} \partial_{x} \omega+\partial_{x} u_{2} \omega=0, & 0<t<T, x \in \mathbb{R}, \\ \omega(0, x)=u_{1,0}(x)-u_{2,0}(x), & x \in \mathbb{R} .\end{cases}
$$


Multiplying (40) by $2 \omega$, an integration on $\mathbb{R}$ gives

$$
\begin{aligned}
\frac{d}{d t}\left\|\partial_{t} \omega(t, \cdot)\right\|_{L^{2}(\mathbb{R})}^{2} & =-2 \int_{\mathbb{R}} u_{1} \omega \partial_{x} \omega d x-2 \int_{\mathbb{R}} \partial_{x} u_{2} \omega^{2} \\
& =2 \int_{\mathbb{R}} \partial_{x} u_{1} \omega^{2} d x-2 \int_{\mathbb{R}} \partial_{x} u_{2} \omega^{2} .
\end{aligned}
$$

Since $u_{1}, u_{2} \in H^{2}(\mathbb{R})$ for every $0 \leq t \leq T$

$$
\left\|\partial_{x} u_{1}\right\|_{L^{\infty}((0, T) \times \mathbb{R})},\left\|\partial_{x} u_{2}\right\|_{L^{\infty}((0, T) \times \mathbb{R})} \leq C .
$$

Therefore, by (41) and (42),

$$
\frac{d}{d t}\|\omega(t, \cdot)\|_{L^{2}(\mathbb{R})}^{2} \leq C\|\omega(t, \cdot)\|_{L^{2}(\mathbb{R})}^{2} .
$$

The Gronwall Lemma and (39) give (5).

\section{Discussion}

The goal of this paper is to investigate the blow-up of the $H^{2}$ norm of the solution of the (inviscid) Burgers equation. The interest for this result is twofold. First of all, we prove an easy to verify relation between the $H^{2}$ norm of the initial datum and the blow-up time for the $H^{2}$-norm of the solution. Moreover, we show a symmetry of the (inviscid) Burgers equation that consists in the invariance of the $H^{2}$ space under the action of that nonlinear equation. Finally, we show that as soon as the solution of the (inviscid) Burgers equation stays in $H^{2}$ is is $L^{2}$ stable with respect to the initial datum. The arguments are based on energy and stability estimates on the the solution of the (viscous) Burgers equation.

Author Contributions: Conceptualization, G.M.C. and L.d.R.; methodology, G.M.C. and L.d.R.; formal analysis, G.M.C. and L.d.R.; investigation, G.M.C. and L.d.R.; resources, G.M.C. and L.d.R.; writing —original draft preparation, G.M.C. and L.d.R.; writing—review and editing, G.M.C. and L.d.R. All authors have read and agreed to the published version of the manuscript.

Funding: G.M.C. has been partially supported by the Research Project of National Relevance "Multiscale Innovative Materials and Structures" granted by the Italian Ministry of Education, University and Research (MIUR Prin 2017, project code 2017J4EAYB and the Italian Ministry of Education, University and Research under the Programme Department of Excellence Legge 232/2016 (Grant No. CUP-D94I18000260001).

Institutional Review Board Statement: Not applicable.

Informed Consent Statement: Not applicable.

Data Availability Statement: Not applicable.

Acknowledgments: G.M.C. is member of the Gruppo Nazionale per l'Analisi Matematica, la Probabilità e le loro Applicazioni (GNAMPA) of the Istituto Nazionale di Alta Matematica (INdAM).

Conflicts of Interest: The authors declare no conflict of interest.

\section{References}

1. Kružkov, S.N. First order quasilinear equations with several independent variables. Math. USSR-Sb. 1970, 81, 228-255. [CrossRef]

2. Bressan, A. The One-Dimensional Cauchy Problem. In Hyperbolic Systems of Conservation Laws; Oxford Lecture Series in Mathematics and Its Applications; Oxford University Press: Oxford, UK, 2000; Volume 20, pp. xii, 250.

3. Dafermos, C.M. Hyperbolic Conservation Laws in Continuum Physics, 4th ed.; Grundlehren der Mathematischen Wissenschaften [Fundamental Principles of Mathematical Sciences]; Springer: Berlin, Germany, 2016; Volume 325, pp. xxxviii, 826. [CrossRef]

4. Holden, H.; Risebro, N.H. Front Tracking for Hyperbolic Conservation Laws, 2nd ed.; Applied Mathematical Sciences; Springer: Heidelberg, Germany, 2015; Volume 152, pp. xiv, 515. [CrossRef]

5. Taylor, M.E. Partial Differential Equations I. Basic Theory, 2nd ed.; Applied Mathematical Sciences; Springer: New York, NY, USA, 2011; Volume 115, pp. xxii+654. [CrossRef] 
6. John, F. Formation of singularities in one-dimensional nonlinear wave propagation. Comm. Pure Appl. Math. 1974, $27,377-405$. [CrossRef]

7. Lax, P.D. Development of singularities of solutions of nonlinear hyperbolic partial differential equations. J. Math. Phys. 1964, 5, 611-613. [CrossRef]

8. Liu, T.P. Development of singularities in the nonlinear waves for quasilinear hyperbolic partial differential equations. J. Differ. Equ. 1979, 33, 92-111. [CrossRef]

9. Coclite, G.M.; Gargano, F.; Sciacca, V. Analytic solutions and singularity formation for the Peakon $b$-family equations. Acta Appl. Math. 2012, 122, 419-434. [CrossRef]

10. Coclite, G.M.; Gargano, F.; Sciacca, V. Up-wind difference approximation and singularity formation for a slow erosion model. ESAIM Math. Model. Numer. Anal. 2020, 54, 465-492. [CrossRef]

11. Kimura, Y. Dynamics of complex singularities for Burgers' equation. In Nonlinear Evolution Equations E Dynamical Systems: NEEDS '94 (Los Alamos, NM); World Scientific Publishing: River Edge, NJ, USA, 1995; pp. 86-93.

12. Coclite, G.M.; Dipierro, S.; Maddalena, F.; Valdinoci, E. Singularity formation in fractional Burgers' equations. J. Nonlinear Sci. 2020, 30, 1285-1305. [CrossRef]

13. Cole, J.D. On a quasi-linear parabolic equation occurring in aerodynamics. Quart. Appl. Math. 1951, 9, 225-236. [CrossRef]

14. Hopf, E. The partial differential equation $u_{t}+u u_{x}=\mu u_{x x}$. Comm. Pure Appl. Math. 1950, 3, 201-230. [CrossRef]

15. Liu, T.P. Hopf-Cole transformation. Bull. Inst. Math. Acad. Sin. 2017, 12, 71-101. [CrossRef]

16. Coclite, G.M.; di Ruvo, L. On classical solutions for a Kuramoto-Sinelshchikov-Velarde-type equation. Algorithms 2020, $13,77$. [CrossRef]

17. Coclite, G.M.; di Ruvo, L. On the well-posedness of a high order convective Cahn-Hilliard type equations. Algorithms 2020, 13, 170. [CrossRef]

18. Coclite, G.M.; di Ruvo, L. A Note on the Solutions for a Higher-Order Convective Cahn-Hilliard-Type Equation. Mathematics 2020, 8, 1835. [CrossRef]

19. Bianchini, S.; Bressan, A. Vanishing viscosity solutions of nonlinear hyperbolic systems. Ann. Math. 2005, 161, 223-342. [CrossRef] 